\title{
La influencia del chat en la escritura de los estudiantes
}

\section{The influence of chat on student writing}

Marcelino Rojas Salazar ${ }^{1}$

Universidad SISE, Lima, Perú

marcelo17comunicacion@gmail.com

\section{RESUMEN}

Cuando se escucha la palabra chat, es muy probable que se la relacione con la tecnología, con la telefonía móvil y con los estudiantes. Sin embargo, es un tipo de comunicación entre las personas, especialmente los jóvenes. Ha tomado importancia, ya que es el medio por el cual los estudiantes expresan cada día sus actividades y sus quehaceres académicos. Es así como en los últimos años se ha tomado como referencia de investigación los aspectos morfológicos, sintácticos y hasta semánticos de este tipo de lenguaje, es decir, la influencia del lenguaje del chat en la escritura de los estudiantes cada día es mayor. A veces se le otorga la responsabilidad a la tecnología de por qué existen tantos cambios en la manera de escribir; también se le responsabiliza al mismo estudiante por su carencia de competencia lingüística en la normativa de la lengua española. No obstante, se debe contar con apoyo de los especialistas para que se pueda aprovechar este lenguaje novedoso en el aprendizaje de los jóvenes y no fatalizar ni limitar la forma creativa de su expresión lingüística. Si bien existe una relevante influencia del uso del lenguaje chat en la escritura formal de los jóvenes estudiantes, ello no significa que se deba erradicar, ya que en los últimos años se ha considerado como una motivación para su desarrollo en las relaciones sociales. Además, los jóvenes deben ser conscientes de la importancia del lenguaje formal y las

1 Magíster en Didáctica de la Comunicación, licenciado en Educación y bachiller en Lingüística de la Universidad Nacional Mayor de San Marcos. Docente de la Facultad de Educación de la Universidad Nacional Mayor de San Marcos, del Área de Humanidades de la Universidad Científica del Sur y del Departamento de Humanidades de la Universidad Privada del Norte. 
diversas formas comunicativas de acuerdo con el contexto, y así se continuará desarrollando la creatividad lingüística en ellos.

\section{PALABRAS CLAVE}

Chat, lenguaje, escritura formal, estudiantes, beneficios, influencia

\section{ABSTRACT}

When it comes to the word chat, it is very likely to be related to technology, mobile phones and students. However, this type of communication between people, especially young people, has taken on importance, since it is the means by which students express their activities and their academic tasks every day. Thus, in recent years the morphological, syntactic and even semantic aspects of this type of language have been taken as a research reference, that is, the influence of the language of the chat in the writing of the students every day is greater. Sometimes the technology is given responsibility for why so many changes in the way of writing, the same student is also held responsible for his lack of linguistic competence in the rules of the Spanish language. However, specialists must be supported in order to take advantage of this new language in the learning of young people and not to fatalize or limit the creative form of their linguistic expression. Although there is a relevant influence of the use of chat language in the formal writing of young students, this does not mean that it should be eradicated, since in recent years it has been considered as a motivation for its development in social relations. In addition, young people must be aware of the importance of formal language and various forms of communication according to the context and thus continue to develop linguistic creativity in them.

\section{KEYWORDS}

Chat, language, formal writing, students, benefits, influence

En el Perú, desde los últimos 20 años, la tecnología referente a los medios de comunicación, así como a los artefactos que llegaron para su respectivo comercio en la ciudad, han avanzado de forma geométrica. Si antes la telefonía fija casera era la panacea para una mejor comunicación 
entre las personas, ahora la telefonía móvil y sus diversas aplicaciones son las que utilizan los jóvenes para conversar, intercambiar diversos archivos, datos, fotografías, enlaces informativos, videos, etc. En la mayoría de esos aspectos se manifiesta un tipo de lenguaje singular con características propias en la escritura, que resulta en ocasiones una manera efectiva para comunicarse $y$, otras veces, una forma distorsionada que altera la lengua española. Por ello, se considera al lenguaje del chat como una influencia relevante en la escritura de la lengua española. Esta situación se manifiesta en los trabajos académicos universitarios, informes, monografías, exámenes y pruebas escritas. De esta forma, habría que buscar un equilibrio entre el uso de este lenguaje y el respeto a las normas propiamente de la lengua española. A continuación, se presentará la definición de chat, luego las posibles formas en que este tipo de lenguaje desea ingresar al mercado literario, después algunas evidencias de investigaciones respecto a la distorsión escrita de la lengua y, finalmente, una reflexión acerca del tema.

El término chat proviene de la lengua inglesa chatter, que se traduce como "conversación o charla". El inicio en el uso se ha presentado desde el propio nacimiento de la red internet en la década de 1970. Chat es una palabra que está definida en el Diccionario de la lengua española (2014) con dos acepciones. La primera lo define como el «intercambio de mensajes electrónicos a través de internet que permite establecer una conversación entre dos o varias personas», y en la segunda lo considera como "servicio que permite mantener conversaciones mediante chats». Ambas coinciden con la forma de establecer un diálogo sobre algún aspecto o temática entre las personas.

Por otro lado, existen tres tipos de chat que permiten dialogar utilizando un dispositivo móvil o una computadora para enviar y recibir mensajes. El primero es el chat basado en la web, que se refiere a los foros virtuales en el que las personas envían y reciben mensajes en tiempo real sobre algún tema específico, como deportes, cine, tecnología, música, moda, programas o series de televisión, etc. El segundo es el chat en tiempo real denominado mensajería instantánea, que ofrece la posibilidad de comunicarse en tiempo real con otras personas que se han agregado al grupo anterior. Para iniciar a chatear, solo se tiene que descargar el software respectivo y crear una cuenta. Los participantes de esta modalidad de chat empiezan su conversación a través de mensajes de texto, videos, imágenes $o$ audios. Algunos ejemplos de mensajería instantánea son el Facebook chat, Windows Messenger, Yahoo Messenger y WhatsApp. El último tipo de chat es el IRC, que pertenece a las siglas Internet Relay Chat, que es la forma más antigua y más conocida de chat que existe. IRC permite la 
comunicación entre dos o más personas conectadas al mismo servidor y salón de chat IRC.

De todos los tipos presentados en los últimos cinco años, el preferido por los jóvenes es el WhatsApp, debido a la efectividad, el costo y la rapidez en los envíos de los mensajes. Si bien lo más importante en una conversación es la comunicación y el entendimiento del mensaje entre las personas, muchas veces, por esa rapidez o por el abuso del lenguaje informal, se llega al extremo de distorsionar la escritura y, por ende, el mensaje de la conversación puede llegar a no comprenderse. Según Cassany (2002, p. 67), expresa lo siguiente:

El lenguaje chat es una expresión relativamente nueva que surge en el contexto de desarrollo de las TIC. Los elementos estructurales del chat son la lista de participantes, el apodo, el perfil, y la conversación que se va generando en pantalla, a medida que cada participante hace una contribución.

Existen otros investigadores que consideran al lenguaje chat como una consecuencia de la utilización de las tecnologías de la información y la comunicación (TIC) en los estudiantes desde la escuela. De igual forma, Cassany manifiesta que una característica particular de este lenguaje del chat es la velocidad en las respuestas llegando al punto de colapsar en mensajes, ya que uno se superpone en otro.

las contribuciones de cada interlocutor aparecen en pantalla a medida que llegan al servidor común, dependiendo de la velocidad de acceso que tenga el ordenador de cada uno o la rapidez con que se tecleen; si también tenemos en cuenta que los interlocutores no pueden usar los sistemas corrientes para marcar fin e inicio de turno (mirada, entonación, movimiento del cuerpo), no es de extrañar que la conversación resultante sea caótica (2002, p. 11).

Por otro lado, se crea una controversia sobre la utilización del lenguaje del chat: si es que conlleva a beneficios o a perjuicios. Cada uno tiene su postura. Es que en realidad no se trata de quién tenga la razón o quién gane, sino de aceptar los hechos y buscar la manera de cómo se pueda solucionar este tipo de fenómeno en la escritura y aprovecharla para un beneficio en la educación de los jóvenes. Así lo manifestó Alonso: «Este fenómeno debe estudiarse desde varias perspectivas. El lenguaje SMS y chat son una nueva forma de comunicación que aprovecha la redundancia del lenguaje escrito a través de las abreviaciones y da forma escrita a la oralidad de la comunicación simultánea» (2008, p. 30).

Si se toman en cuenta los aspectos positivos de desarrollar este tipo de lenguaje, uno de los beneficios será desarrollar la participación activa de 
la conversación entre los estudiantes que presentan timidez o son retraídos en una escuela o universidad, al punto de ni siquiera decir su nombre en público. Otro beneficio será el fortalecimiento de valores que tengan acceso a otros principios, como la confianza y el respeto a la opinión de los demás, ya que se establece una relación de amistad y de confianza al manejar el mismo código que el joven estudiante comparte con el profesor. De igual forma, otro beneficio consistirá en entablar una conversación simultánea con varias personas en el mundo sin barreras territoriales, culturales ni temporales. Finalmente, existe el beneficio de ayudar a formar mejores jóvenes que serán profesionales con una conciencia ética y honesta.

Por ejemplo, en el diario El Heraldo de Colombia se publicó uno de los fragmentos de la adaptación para smartphones de El ingenioso hidalgo don Quijote de la Mancha, que forma parte de un proyecto denominado Clásicos traducidos al lenguaje chat.

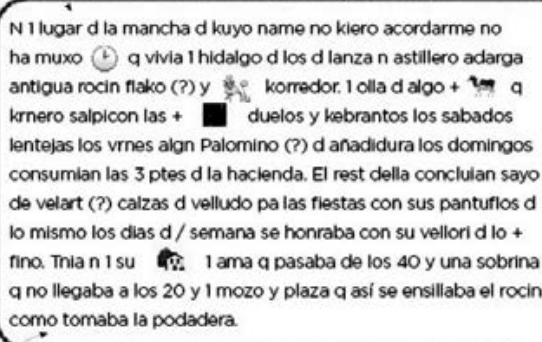

FIGURA 1. El ingenioso hidalgo don Quijote de la Mancha (El Heraldo, 2016)

Original: primer párrafo del Quijote. Un lugar de la Mancha, de cuyo nombre no quiero acordarme, no ha mucho tiempo que vivía un hidalgo de los de lanza en astillero, adarga antigua, rocín flaco y galgo corredor. Una olla de algo más vaca que carnero, salpicón las más noches, duelos y quebrantos los sábados, lentejas los viernes, algún palomino de añadidura los domingos, consumían las tres partes de su hacienda. El resto della concluían sayo de velarte, calzas de velludo para las fiestas con sus pantuflos de lo mismo, los días de entre semana se honraba con su vellorí de lo más fino. Tenía en su casa una arma que pasaba de los cuarenta, y una sobrina que no llegaba a los veinte, y un mozo de campo y plaza que así ensillaba el rocín como tomaba la podadera.

Una forma de motivar a los adolescentes y jóvenes en la lectura sería abordar las novelas, cuentos u otros textos en el lenguaje del que ellos están familiarizados. Otro ejemplo se presenta para una novela de Gabriel García Márquez. 


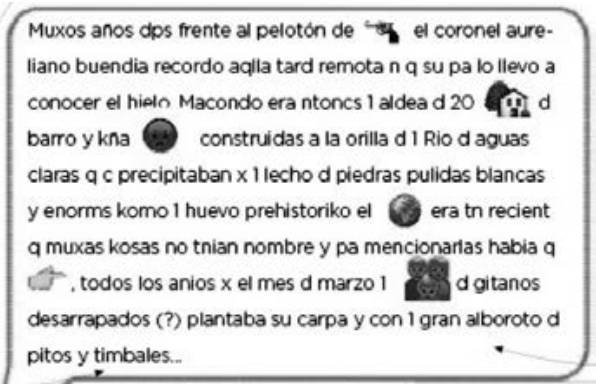

FIGURA 2. Cien años de soledad (EI Heraldo, 2016)

Original: primer párrafo de Cien años de soledad. Muchos años después, frente al pelotón de fusilamiento, el coronel Aureliano Buendía había de recordar aquella tarde remota en que su padre lo llevó a conocer el hielo. Macondo era entonces una aldea de veinte casas de barro y cañabrava construidas a la orilla de un río de aguas diáfanas que se precipitaban por un lecho de piedras pulidas, blancas y enormes como huevos prehistóricos. El mundo era tan reciente que muchas cosas no tenían nombre y para mencionarlas había señalarlas con el dedo. Todos los años, por el mes de marzo, una familia de gitanos desarrapados plantaba su carpa cerca de la aldea y con un gran alboroto de pitos y timbales...

Como se pudo observar, en ambos fragmentos, existe una simplificación de las palabras. Además, se complementa de unas figuras Ilamadas emojis. Es decir, ante la problemática de la falta de lectura o la carencia de hábitos de lectura, la tecnología en los teléfonos móviles ha tomado la iniciativa de acercar al estudiante a un mundo literario donde está inmerso el lenguaje que utiliza de forma cotidiana. Sin embargo, no se debe confundir la comprensión de textos con la redacción de textos. Si bien ambos presentan una relación lingüística, pertenecen a diferentes planos.

La investigadora Heidy Robles, docente de la Universidad del Norte en Barraquilla, comenta que los jóvenes se sienten atraídos por este lenguaje del chat porque se ahorran tiempo. No obstante, aclara que la labor de los profesores es concientizar al estudiante de que existen contextos y tiempos para escribir de esta manera. «Es decir que, si yo debo escribir un informe o una carta, debo utilizar un registro de la lengua formal [...] No podemos impedir a los jóvenes que hagan uso de ese tipo de abreviaturas o términos, pero que solo lo hagan en el chat».

Claro está que no se debe confundir el chat, que es una herramienta de socialización y mensajería instantánea que puede realizarse en los equipos de telefonía celular como en las computadoras, con el concepto de lenguaje del chat, que es el tipo de escritura con características propias de aquel sistema, como la presencia de abreviaturas de manera indiscri- 
minada, la repetición de consonantes o vocales, la falta de tilde en las palabras, la omisión del uso de grafías como $h, z, c$, o la omisión de utilizar los signos de puntuación. Aquellas características se pueden observar en la siguiente figura.

\begin{tabular}{|c|c|}
\hline Abreviatura: & Significedos \\
\hline 100 pre & siempine \\
\hline aki & aquis \\
\hline BFV & 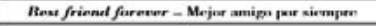 \\
\hline ba & bisen \\
\hline DM & Direct message - Mensaje difecto o mensaje private \\
\hline dred & donde \\
\hline $\mathbf{F B}$ & Vinceloook \\
\hline FYIIPT & 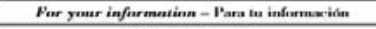 \\
\hline IIX & I lowe you - te anes \\
\hline $\mathbf{J K} \in \mathbf{J} / \mathbf{K}$ & Juse Nieldieng - nele brosecando \\
\hline 101. & Laurfhing eet loud - Kiencls a carcajadas \\
\hline NP & No problem - no hay problecma \\
\hline Novans dyp & 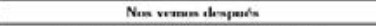 \\
\hline NVM & Newerming - OAvílales, no te preocupos \\
\hline Pa q & para que \\
\hline$m$ & paes \\
\hline RT & Re-Thenet \\
\hline Salu2 & Sulucters \\
\hline ntoy / ala & cotog / collit \\
\hline $\mathrm{tb} / \mathrm{tmb}$ & tambien \\
\hline $\operatorname{Thx} / \operatorname{Tax} / \operatorname{Man} x$ & Thanks-gracias \\
\hline xfin & Purfa - por faver \\
\hline
\end{tabular}

FIGURA 3. Abreviaturas utilizadas en las redes sociales (Eres y Almeida, 2009)

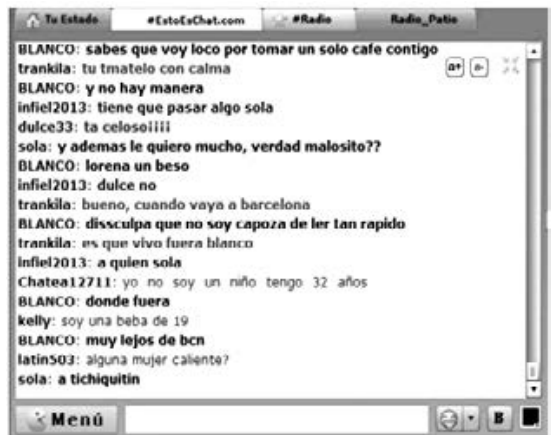

FIGURA 4. Conversación chat (Martínez, 2014, p. 58)

\begin{tabular}{|c|}
\hline Etstofechat.com \\
\hline 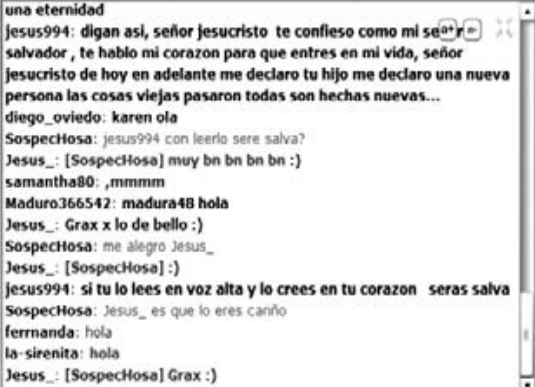 \\
\hline
\end{tabular}

FIGURA 5. Conversación chat (Martínez, 2014, p. 61) 
En las figuras 4 y 5 se puede observar una conversación utilizando una sala de chat. Se presenta una afinidad amical y, por ende, al momento de escribir se produce el lenguaje chat donde se omite la tilde, se utiliza la letra mayúscula de manera ocasional, se duplican algunas consonantes, se escriben los signos de exclamación y de interrogación al final pero no al inicio, se abrevian algunas palabras eliminando las vocales en su totalidad y se reemplaza una consonante por palabras. Esta situación ocurre de forma natural y cotidiana en los jóvenes estudiantes.

Asimismo, se debe precisar que el lenguaje formal se refiere a un lenguaje utilizado en situaciones donde se requiere un protocolo. Aquí prima una correcta pronunciación, un adecuado vocabulario y una correcta estructura de las oraciones. Se evitan las muletillas, vulgarismos, modismos y jergas. Todo ello se evidencia cuando el estudiante escribe o expone sus ideas frente a una situación académica. No obstante, lo opuesto de aquello será la informalidad del lenguaje. Esto no implica a lo grosero, ni mucho menos a las lisuras o palabras soeces que llegan a ofender o herir a las personas. Por el contrario, la informalidad en el lenguaje está relacionada con el uso de la lengua de manera libre, sin barreras normativas, donde la persona se expresa con familiaridad en un plano coloquial con amigos o personas que tiene estima. Esta es la situación que ha resultado en el lenguaje del chat, un claro ejemplo donde priman los sentimientos y no se juzga por dejar de utilizar la norma de ortografía. En la investigación de Castro (2014, p. 12) se manifiesta lo siguiente:

El aula, después de la familia, es el lugar más importante para el alumnado, pues ahí establece vínculos sociales, afectivos y significativos, y ahí pueden estar las herramientas necesarias para su aprendizaje sistemático. Es el ambiente del proceso de enseñanza-aprendizaje, donde se adquiere, produce y reproduce el conocimiento.

Es decir, el estudiante, al estar vinculado con sus compañeros y amigos de la escuela, desarrollará el lenguaje informal propiamente dicho, mediante la tecnología de un teléfono móvil o computadora, para elaborar sus respectivos trabajos, proyectos o quehaceres académicos.

En el Perú, un caso particular en el que se investigó sobre la influencia del chat en la escritura de los estudiantes fue realizado por Martínez, quien analizó la escritura de 80 estudiantes de una escuela de nivel secundario en el distrito de Carabayllo, en Lima, mediante la aplicación de un cuestionario de preguntas abiertas donde cada uno respondió lo que se planteó. Es así como expresa la idea siguiente:

El uso constante y regular de programas de mensajería, foros y páginas web interactivas exige mensajes breves, rápidos y sin formalis- 
mos [...] En estos contextos comunicativos, la prioridad de los participantes es mantener una comunicación fluida a través de contenidos básicos. Todo esto genera un tipo de lenguaje que se caracteriza por una pobreza léxica y abandono de las reglas ortográficas. A través del uso constante, los adolescentes internalizan el lenguaje chat y lo suelen emplear con regularidad en sus sesiones de chat $(2014$, p. 91$)$.

En la figura 6 se puede observar la manera en que los estudiantes elaboran sus propios signos para representar a las palabras.

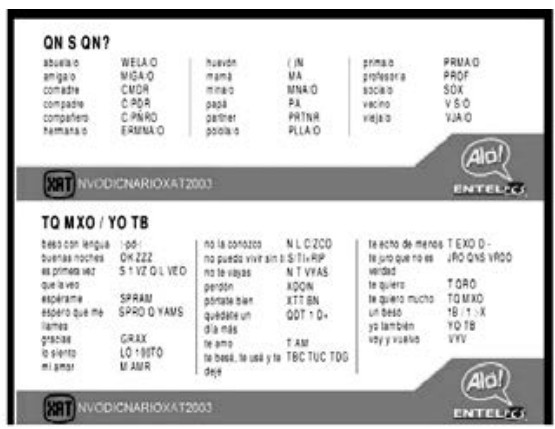

FIGURA 6. Léxico de chat (Martínez, 2004, p. 46)

Aquí se observan las palabras que forman parte del seudónimo o el llamado nickname y la conversación escrita, donde se evidencia la repetición de grafías varias veces, la falta de uso de mayúsculas y la carencia de aplicar la tilde.

Así también, la investigación de Rocha (2006), de la Facultad de Letras y Comunicación de la Universidad de Colima en Colombia, concluyó que «el chat es un sistema sincrónico en red cada vez más utilizado, cuyo lenguaje se impone poco a poco al interior de la misma comunidad». Como menciona la investigadora colombiana, este tipo de lenguaje se viene «imponiendo» en la redacción. ¿Qué medidas se podría tomar para controlar esta situación? ¿Se debe eliminar el chat?

En un artículo publicado por Consuelo Rehbein, se explicaba que la masificación de los teléfonos celulares ha provocado el lenguaje chat o ciberlenguaje que es utilizado en los portales de internet, redes sociales y en los mensajes SMS. Asimismo, este lenguaje del chat presenta características y rasgos propios de una economía o simplificación en el lenguaje formal, es decir, no se cumple con las normas de abreviación propiamente dicha. Por ello, el uso excesivo de este lenguaje chat en los documentos es considerado informal. La periodista realiza una compilación de los principales términos que se utiliza en la escritura chat. 


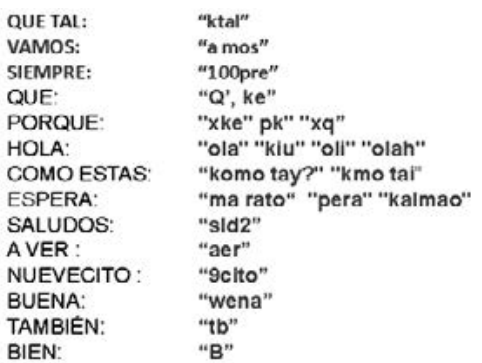

FIGURA 7. Repertorio léxico (Rocha, 2006, p. 46)

Se observa que la figura 7 presenta una forma de simplificación por parte del lenguaje chat. Se reducen las vocales para que solo se visualicen las consonantes.

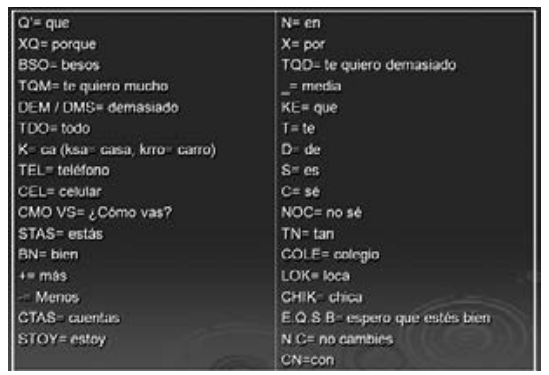

FIGURA 8. Repertorio léxico (Rocha, 2006, p. 46)

En la figura 8, aparece una forma de representación de estados de ánimo mediante letras duplicadas.

Sin embargo, existen algunos simpatizantes en el uso de este tipo de lenguaje, como Slotniski (2015), quien afirma que la Universidad de Alberta en Estados Unidos encontró que el lenguaje utilizado por los jóvenes en los mensajes instantáneos y correos electrónicos no afecta la ortografía de los estudiantes. Esta sentencia se produjo de acuerdo con la investigación basada en 40 estudiantes de 12 a 17 años de edad, que considera que una posible solución a esta situación podría ser aggiornando la ortografía al máximo, de tal modo que la lengua sea más eficaz y eficiente. Es decir, que las reglas ortográficas sean pocas, claras y sin excepciones. Por ejemplo, que no haya letras mudas (como la $h$ ) o evitar las consonantes que suenen igual aunque se escriban diferente, una superposición de letras (como sucede con la $v$ y la $b$ ). Esta situación se asemeja a la reflexión que planteó Gabriel García Márquez con «Botella al mar para el dios de las palabras» en el Congreso Internacional de la Lengua Española en Zacatecas, en 1997. García Márquez explicaba allí que la ortografía debería simplificarse para darle una mayor libertad en términos de escribir sin restricciones ortográficas. 
La mayoría de investigadores concuerdan en que el uso del lenguaje del chat por los estudiantes se debe a que manifiestan un tipo de relación amical y de confianza en el que se explayan sin restricción ni forma. Ello no implica que los adolescentes o jóvenes desconozcan la normativa de la lengua española, ya que la misma convivencia en la escuela o universidad hace que estén inmersos en la formalidad de la escritura. Sin embargo, parece que aquello les resulta irrelevante.

En una encuesta realizada en 2013, en la ciudad de La Dorada Caldas, se preguntó a un grupo de 97 estudiantes sobre por qué los alumnos escriben en el chat con mala ortografía. Los resultados fueron: porque no saben $(11 \%)$, porque no quieren (38\%), por la rapidez $(28 \%)$ y por la originalidad (23\%).

Si se analizan las respuestas, se observa que el $89 \%$ de estudiantes consideran que se escribe con errores en el chat de forma intencional; en otras palabras, es un acto de rebeldía, de originalidad, de creatividad o de sencillez.

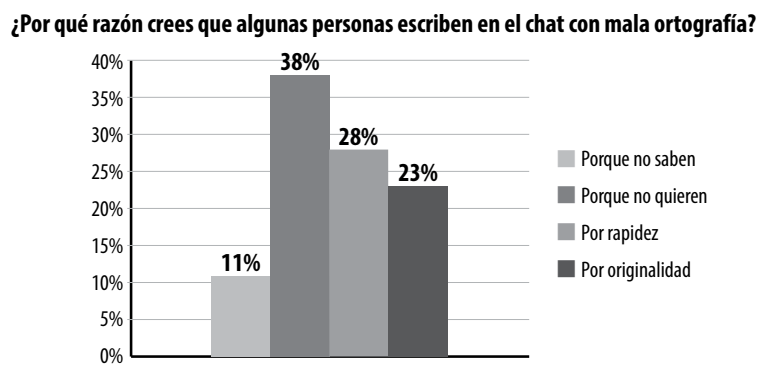

FIGURA 9. Causas de la escritura con mala ortografía (Gonzáles, 2014)

En conclusión, si bien el lenguaje de chat se puede considerar como una forma creativa, elaborar esos tipos de signos que representan palabras y a veces frases ayuda al desarrollo social de las personas con timidez o temor al expresarse en público. Asimismo, motiva el hábito de la lectura, transformando textos o novelas clásicas a este tipo de lenguaje. No se debe confundir con el uso formal de la lengua española en un contexto del quehacer académico en la escuela o en la universidad.

A pesar de las ventajas que se pueda proponer, existe una alta influencia del lenguaje chat en la escritura formal de los jóvenes estudiantes. Si se continúa utilizando este lenguaje sin ningún control, podrían producirse efectos que no solo distorsionen la escritura, sino el mensaje de un texto. Por ello, se recomienda que los docentes y los especialistas expliquen con suma claridad este fenómeno lingüístico y concienticen a los estudiantes para ser competentes lingüísticamente. Es decir, el estudiante debe 
manejar los diversos contextos comunicativos para desarrollar y utilizar el tipo de lenguaje adecuado en el que se sientan más cómodos. Pueden seguir utilizando el lenguaje del chat en sus redes sociales en la computadora o en el teléfono móvil, pero deben evitarlo en contextos formales y con mayor razón en los trabajos académicos. 


\section{REFERENCIAS BIBLIOGRÁFICAS}

ALONSO, E. y Perea, M. (2008). SMS: Impacto social y cognitivo. Valencia: Editorial Escritos de Psicología.

CASTRO, M. (2014). Variaciones de lenguaje (formal e informal) en el contexto educativo en la ciudad de Tefé (Amazonas, BR): ¿Diversidad o fracaso escolar? (tesis de doctorado). Universidad de Valladolid, Valldolid. Recuperado de https://uvadoc.uva.es/bitstream/10324/4577/1/TESIS507-140307.pdf

CASSANY, D. (2002). La alfabetización digital. Recuperado de http://www. estrategiaeducativa.com.mx/masterconsecuencias/Cassany.pdf

El Heraldo. (23 de abril de 2016). El lenguaje chat "rejuvenece", pero supone riesgos al idioma. Recuperado de https://www.elheraldo.co/entretenimiento/el-lenguaje-chat-rejuvenece-pero-supone-riesgos-al-idioma-256388

ERES, I. y ALMEIDA, P. (2009). Un estudio sobre los cambios lingüísticos del español escrito en charlas informales por internet. Recuperado de http:// www.scielo.br/pdf/tla/v48n1/11.pdf

KHELFA, M. (2015). El chat como estrategia para fomentar el aprendizaje de los estudiantes hispanistas argelinos. Recuperado de http://dspace.univtlemcen.dz/bitstream/112/8153/1/khelfa-mohamed.pdf

MARTÍNEZ, D. (2014). El lenguaje chat y redacción escolar en adolescentes de Lima (tesis de licenciatura en Lingüística). Universidad Nacional Mayor de San Marcos, Lima.

Real Academia Española. (2001). Diccionario de la lengua española, Madrid: Espasa Calpe.

ROCHA, M. (2006). El lenguaje de los jóvenes del chat. Recuperado de http:// bibliovirtual.upn.edu.pe:2153/lib/upnortesp/detail.action?docID=10125 $348 \& \mathrm{p} 00=\mathrm{el}+\mathrm{chat}$

ROQUET, G. (2004). Los chaty su uso en educación. Recuperado de http://tic. sepdf.gob.mx/micrositio/micrositio2/archivos/ChatsYUsoEducativo.pdf

SLOTNISKY. D. (28 de mayo de 2015). Cómo influyen las nuevas tecnologías en la escritura. Recuperado de https://www.publimetro.cl/cl/tecnologia/2017/02/27/guia-chat-chile.html

TIRIRA, M. (2013). La escritura en las redes sociales. Recuperado de http:// www.usfq.edu.ec/publicaciones/para_el_aula/Documents/para_el_ aula_08/pea_008_0010.pdf 